\title{
Strongyloidiasis as a Cause of Chronic Diarrhea, Identified Using Next-Generation Strongyloides stercoralis-Specific Immunoassays
}

\author{
Alisa Thamwiwat • Rojelio Mejia • Thomas B. Nutman • \\ Jeffrey T. Bates
}

Published online: 6 July 2014

(C) Springer International Publishing AG (outside the USA) 2014

\begin{abstract}
A 63-year-old-male Vietnam War veteran noted a 6-month history of watery diarrhea. A colonoscopy 6 months prior to presentation was significant for colonic mucosa with inflammation, sheets of eosinophils, and an acellular structure, which was suspicious for a parasite. Initial laboratory testing on presentation revealed an elevated eosinophil count, prompting an investigation for a parasitic infection. Three stool microscopy examinations were negative for parasite ova and larvae, but the patient had a positive Strongyloides immunoglobulin-G antibody on an enzyme-linked immunosorbent assay (ELISA). Confirmation of $S$. stercoralis infection was achieved using a Strongyloides-specific luciferase immunoprecipitation systems (LIPS) assay. Accurate diagnosis of Strongyloides infection is particularly crucial for patients who are currently immunocompromised or will be
\end{abstract}

A. Thamwiwat $\cdot$ J. T. Bates

Department of Medicine, Baylor College of Medicine,

Houston, TX, USA

A. Thamwiwat

e-mail: thamwiwa@bcm.edu

\section{R. Mejia}

Laboratory of Clinical Parasitology and Diagnostics, National School of Tropical Medicine, Baylor College of Medicine, Houston, TX, USA

e-mail: Rojelio.Mejia@bcm.edu

R. Mejia $\cdot$ T. B. Nutman

Helminth Immunology Section, Laboratory of Parasitic Diseases, National Institute of Allergy and Infectious Diseases, National Institutes of Health, Bethesda, MD, USA

T. B. Nutman

e-mail: tnutman@niaid.nih.gov

J. T. Bates $(\bowtie)$

Medical Care Line, Michael E. DeBakey VA Medical Center,

Houston, TX, USA

e-mail: jeffreyt.bates@va.gov immunosuppressed with medications, as immunosuppression can lead to accelerated autoinfection and systemic dissemination, which, if untreated, carries a fatality rate of nearly $100 \%$.

Keywords Strongyloides · Diagnostic $\cdot$ Eosinophilia · Diarrhea $\cdot$ Tropical medicine $\cdot$ Parasitic infection

\section{Introduction}

\section{Case Presentation}

A 63-year-old-male Vietnam War veteran noted a 6-month history of watery diarrhea. He was admitted to the hospital for volume depletion. There were no documented fevers. He denied hematochezia, melena, constipation, nausea, vomiting, abdominal pain, night sweats, and weight loss. The patient had had no recent travels, but he had been in Vietnam for 1 year in 1970. The patient's abdominal examination was unremarkable. Laboratory values were significant for an elevated white blood cell count of $11.4 \mathrm{~K} / \mathrm{mm}^{3}$ and an initial absolute eosinophil count of $1,254 / \mathrm{mm}^{3}$ (normal range 0-400/ $\mathrm{mm}^{3}$ ), which rose to $2,173.6 / \mathrm{mm}^{3}$ during hospitalization. The patient had had a colonoscopy 6 months prior to admission because of a positive fecal occult blood test (FOBT). Biopsy showed colonic mucosa with inflammation, sheets of eosinophils, and an acellular structure surrounded by eosinophils, which was felt to be suspicious for a parasite (Fig. 1). This information, combined with the peripheral eosinophilia, prompted evaluation for a parasitic infection. The patient's stool was negative for microsporidia on trichrome blue stain, Cyclospora/Isospora/Cryptosporidium on acid-fast stain, Cryptosporidium antigen, Giardia lamblia enzyme immunoassay (EIA), Shigella/Salmonella/Campylobacter culture, Yersinia culture, and Clostridium difficile polymerase chain reaction (PCR). His serum was negative for antibodies to 


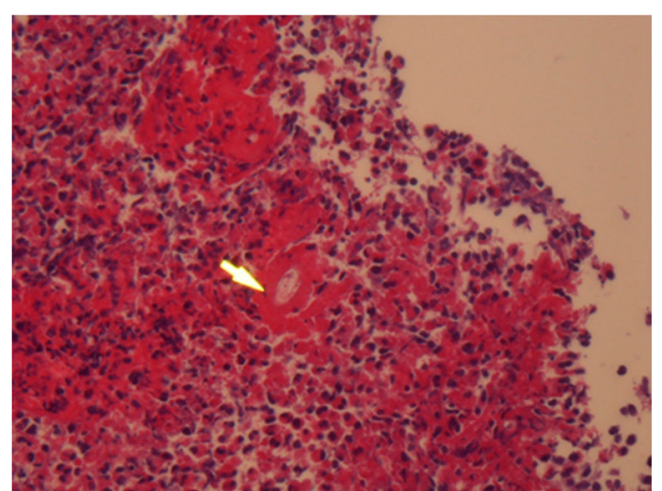

Fig. 1 Image of the colon biopsy from the patient, obtained 6 months prior to admission. It contains fragments of colonic mucosa that have inflammation and sheets of eosinophils. There is also an acellular structure, surrounded by eosinophils, which is suspicious for a parasite (white arrow) [400×, hematoxylin and eosin stain]

Entamoeba histolytica and Cryptococcus. A screening chemiluminescence immunoassay for human immunodeficiency virus (HIV) was negative. Three stool microscopy examinations were negative for parasite ova and larvae. The patient was found to have a positive Strongyloides immunoglobulin (Ig)-G antibody on an enzyme-linked immunosorbent assay (ELISA) performed commercially (LabCorp, Houston, TX, USA). Subsequently, the patient's blood was sent to the Laboratory of Parasitic Diseases, National Institute of Allergy and Infectious Diseases (NIAID) at the National Institutes of Health (NIH) for additional testing with the NIE-based luciferase immunoprecipitation systems (LIPS) assay and stool multi-parallel quantitative PCR (qPCR). The Strongyloidesspecific LIPS assay result was markedly positive for Strongyloides. A single stool collected after the colonoscopy was negative for Strongyloides, using qPCR. The patient was prescribed ivermectin $200 \mu \mathrm{g} / \mathrm{kg} /$ day for 2 days. Two weeks post-discharge, his eosinophilia had decreased to $869 / \mathrm{mm}^{3}$. Two months following hospital discharge, it had decreased to $328 / \mathrm{mm}^{3}$, and his diarrhea had resolved. It has not returned now 16 months since his initial treatment.

\section{Discussion}

Infection with Strongyloides stercoralis occurs when the worm burrows through exposed skin or when it is swallowed [1]. Although symptoms can include pruritus, cough, dyspnea, diarrhea, and abdominal pain [2], patients can also remain asymptomatic. Autoinfection, the process by which Strongyloides can complete its entire life cycle in the human host, may permit the parasite to persist in the host for decades. Thus, clinical manifestations can occur long after the initial infection [3]. This patient likely contracted the infection in Vietnam over 40 years ago and remained asymptomatic until 6 months prior to hospital admission.
Invasive strongyloidiasis is endemic in the tropical and subtropical areas of Southeast Asia and Africa [4]. It is uncommon in the USA, with a prevalence of approximately $4 \%$, concentrated in the eastern areas of the country [5]. For those patients diagnosed in North America and Western Europe, the infection is most commonly found in immigrants from endemic areas $[2,4,6]$.

The sensitivity of detecting Strongyloides in the stool by microscopy (and related techniques) is low, estimated at only $50 \%$ with three samples [5]. Although a crude soluble somatic Strongyloides-based IgG ELISA can be positive, the positive test can occasionally be a result of cross-reactivity to other helminths, particularly filariae. Thus, this patient's blood and stool were sent to the Laboratory of Parasitic Diseases, NIAID, NIH for confirmation with both a newer LIPS assay $[7,8]$ and qPCR for Strongyloides [9]. Both tests are known to be more sensitive and specific than the stool microscopy, and neither has cross-reactivity with other helminth infections [7-9]. qPCR is more sensitive than stool microscopy, but it requires the parasite or its DNA to be present in a small volume of stool (typically $50 \mathrm{mg}$ ). With the intermittent ova/ larvae shedding of Strongyloides, qPCR may be falsely negative because of stool sampling errors. Serology with the LIPS assay has the highest sensitivity of the three tests, estimated at about $97 \%$ or greater [7, 8]. Accurate diagnosis of Strongyloides infection is particularly crucial for patients who are immunocompromised because of illness or immunosuppressant medications. Immunosuppression can lead to disseminated disease and hyperinfection syndrome, which, untreated, carries a nearly $100 \%$ mortality rate [10].

Currently, the treatment of choice for strongyloidiasis is ivermectin, although albendazole can be used as an alternative [11]. In patients with a positive stool examination prior to treatment, repeat stool examinations should be performed between 2 and 6 weeks after treatment, to ensure eradication of the parasite $[11,12]$. In those with negative stool examinations, either decreasing antibody levels or a reduction in peripheral eosinophilia can be used to assess treatment success indirectly. One retrospective study, which included 31 patients, noted that responders to therapy had a significant reduction in the peripheral eosinophil count from a mean of 1,173 cells $/ \mu \mathrm{L}$ to 500 cells $/ \mu \mathrm{L}$ after an average of 96 days, and they had a reduction in the serologic IgG titer by $\sim 270$ days [12]. Since our patient's stool samples were initially negative for Strongyloides, resolution of his symptoms and the peripheral eosinophilia were monitored to document cure, albeit indirectly.

Clinicians must consider invasive strongyloidiasis in patients with chronic diarrhea and peripheral eosinophilia, especially in people who have lived in or traveled to endemic areas. Clinicians must also be aware of the possibility of autoinfection, which may continue for decades prior to clinical presentation $[2,6]$. Accurate diagnosis is critically 
important in patients who are or will be immunocompromised, because of the possibility of hyperinfection syndrome and disseminated disease. Negative stool microscopy examinations should not exclude the diagnosis of strongyloidiasis, and the newer-generation immunoassays and/or qPCR can identify Strongyloides infection with greater sensitivity and specificity than the current standard tests.

Acknowledgments Dr. Jennifer Cowart was of great assistance in the review of this manuscript. Support for preparation of this work was provided by the Department of Veterans Affairs.

\section{Compliance with Ethics Guidelines}

Conflict of Interest Alisa Thamwiwat, Rojelio Mejia, Thomas B. Nutman, and Jeffrey T. Bates declare that they have no conflict of interest.

Human and Animal Rights and Informed Consent This article does not contain any studies with human or animal subjects performed by any of the authors.

\section{References}

1. Zeehaida M, Zairi NZ, Rahmah N, Maimunah A, Madihah B. Strongyloides stercoralis in common vegetables and herbs in Kota Bharu, Kelantan. Malaysia Trop Biomed. 2011;28(1):188-93.

2. Pelletier Jr LL, Gabre-Kidan T. Chronic strongyloidiasis in Vietnam veterans. Am J Med. 1985;78(1):139-40.

3. Hauber HP, Galle J, Chiodini PL, Rupp J, Birke R, Vollmer E, et al. Fatal outcome of a hyperinfection syndrome despite successful eradication of Strongyloides with subcutaneous ivermectin. Infection. 2005;33(5-6):383-6.
4. Dawson-Hahn EE, Greenberg SL, Domachowske JB, Olsen GB. Eosinophilia and the seroprevalence of schistosomiasis and strongyloidiasis in newly arrived pediatric refugees: an examination of Centers for Disease Control and Prevention screening guidelines. J Pediatr. 2010;156(6):1016-8. 1018.e1.

5. Boulware DR, Stauffer WM, Hendel-Paterson BR, Rocha JLL, Seet $\mathrm{RC}$, Summer AP, et al. Maltreatment of Strongyloides infection: case series and worldwide physicians-in-training survey. Am J Med. 2007;120(6):545.e1-8.

6. Pelletier Jr LL. Chronic strongyloidiasis in World War II Far East exprisoners of war. Am J Trop Med Hyg. 1984;33(1):55-61.

7. Ramanathan R, Burbelo P, Groot S, Iadarola MJ, Neva FA, Nutman TB. A luciferase immunoprecipitation systems assay enhances the sensitivity and specificity of diagnosis of Strongyloides stercoralis infection. J Infect Dis. 2008;198:444-51.

8. Krolewiecki AJ, Ramanathan R, Fink V, McAulife I, Cajal SP, Won $\mathrm{K}$, et al. Improved diagnosis of Strongyloides stercoralis using recombinant antigen-based serologies in a community-wide study in northern Argentina. Clin Vaccine Immunol. 2010;17(10):1624-30.

9. Mejia R, Vicuña Y, Broncano N, Sandoval C, Vaca M, Chico M, et al. A novel, multi-parallel, real-time polymerase chain reaction approach for eight gastrointestinal parasites provides improved diagnostic capabilities to resource-limited at-risk populations. Am J Trop Med Hyg. 2013;88(6):1041-7.

10. Mejia R, Nutman TB. Screening, prevention and treatment for hyperinfection syndrome and disseminated infections caused by Strongyloides stercoralis. Curr Opin Infect Dis. 2012;25(4):458-63.

11. Suputtamongkol Y, Premasathian N, Bhumimuang K, Waywa D, Nilganuwon S, Karuphong E, et al. Efficacy and safety of single and double doses of ivermectin versus 7-day high dose albendazole for chronic strongyloidiasis. PLoS Negl Trop Dis. 2011;5(5):e1044.

12. Nuesch R, Zimmerli L, Stockli R, Gyr N, Hatz CFR. Imported strongyloidosis: a longitudinal analysis of 31 cases. J Travel Med. $2005 ; 12: 80-4$. 